\title{
ESTUDIO DE ALTERACIONES PROVOCADAS POR LABOREO AGRÍCOLA SOBRE CONJUNTOS CERÁMICOS EN AGUA AMARGA (TUPUNGATO, MENDOZA, ARGENTINA)
}

\author{
STUDY OF DISTURBANCE INDUCED BY TILLAGE ON CERAMIC \\ ASSEMBLAGES IN AGUA AMARGA (TUPUNGATO, MENDOZA, ARGENTINA)
}

\author{
María José Ots ${ }^{1}$
}

\begin{abstract}
En este trabajo proponemos identificar los efectos de la labranza mecánica en el sitio arqueológico Agua Amarga, ubicado en una finca frutivitivinícola en el Valle de Uco, Mendoza (República Argentina). Con el objetivo de evaluar la alteración de la distribución espacial y la representatividad de los conjuntos cerámicos comparamos las muestras obtenidas mediante la recolección sistemática e intensiva de superficie y la excavación de sondeos de control. La metodología del trabajo incluye el mapeo de la distribución de la cerámica, análisis estadístico de distribución de puntos, identificación de clases de artefactos mediante el análisis tecnotipológico de la cerámica y de la representatividad de dichas clases en la superficie mediante pruebas estadísticas. Teniendo en cuenta el tipo y la frecuencia de las operaciones agrícolas desarrolladas en el sitio, comentamos los resultados en relación con fenómenos asociados a la formación del registro como son el efecto dimensional y la perturbación ocupacional. Demostramos que el conjunto cerámico estudiado, compuesto por tres tipos característicos del Centro Oeste argentino, es representativo de las ocupaciones del sitio, concluyendo que algunos aspectos (cronológicos, tecnológicos) pueden ser resueltos en los sitios ubicados en zonas aradas a pesar de la modificación a la que se encuentran sometidos.
\end{abstract}

Palabras claves: arqueología de zonas aradas, arqueología superficial, cerámica.

The purpose of this work is to identify the effects of mechanical tillage on the archaeological site Agua Amarga located in Valle de Uco (Mendoza, Argentina). In order to evaluate disturbances in the spatial distribution and representability of ceramics assemblages, we compared samples obtained by systematic and intensive surface collection with those from controlled test excavations. The working methodology included mapping of ceramics distribution, statistical analysis of point distribution, artifact identification by technical-typological analysis of ceramics, and representability analysis of these artifacts on the surface by statistical tests. Finally, taking into account the kind and frequency of the agricultural activities, we discuss the results of the study in relation to phenomena like size effect and occupational disturbance. We demonstrate that the ceramic assemblage studied, composed of three characteristic wares or type series of West-Central Argentina, is representative of site occupations, concluding that some aspects (such as chronological and technological factors) can be resolved even in surface collections from sites disturbed by plowing.

Key words: Plow-zone archaeology, surface archaeology, pottery.

En el contexto de las investigaciones históricas y arqueológicas de la dominación incaica en el Centro Oeste argentino $^{1}$, abordamos el estudio del sitio Agua Amarga (Valle de Uco, Mendoza), con el objetivo de determinar las características y los alcances de la presencia incaica en el área². Por el momento, la cerámica constituye para este sector el principal indicador de dicha ocupación, ante la ausencia de restos arquitectónicos o de la red vial.

En el caso que presentamos, la posibilidad de determinar áreas de actividad, patrones de asentamiento y de uso del espacio se encuentra limitada por las condiciones del sitio, ubicado en un espacio sometido a la explotación agrícola. Estas actividades modifican también las condiciones de conservación de materiales frágiles como la cerámica, por lo cual las conclusiones que se establezcan a partir de estos indicadores deberán considerar el nivel en que las muestras son representativas de los componentes originales.

En este trabajo proponemos la identificación del grado de alteración del sitio y las consecuencias en la representatividad de la alfarería recolectada en muestreos superficiales sistemáticos y sondeos. De acuerdo con estos objetivos, hemos adoptado una metodología de trabajo para registrar y analizar los datos adecuada a las condiciones del sitio.

\footnotetext{
Unidad de Antropología, INCIHUSA, CONICET. CRICYT, Casilla de Correo 131 (5500) Mendoza, Rca. Argentina. mjots@lab.cricyt.edu.ar
} 


\section{Ubicación y Descripción del Sitio}

La subárea arqueológica Centro Oeste argentino se extiende entre los ríos Jachal-Zanjón en la provincia de San Juan y Diamante en la de Mendoza. Este territorio conforma una unidad ecológica y cultural, incluida dentro del proceso prehistórico del Área Andina meridional. Ambientalmente se caracteriza como un "territorio continental, de clima árido a semiárido, cuyo promedio de precipitaciones no sobrepasa los $200 \mathrm{~mm}$ anuales" (Prieto 2000:29). Las poblaciones locales del momento tardío, Huarpes, ocuparon tres tipos ecológicos: la montaña, el piedemonte y la llanura. Estos grupos fueron conquistados respectivamente por incas y españoles.

Los conos aluviales de los ríos Tunuyán y de las Tunas han formado uno de los oasis más ricos de la provincia de Mendoza, en el Valle de Uco. En esta área se encuentra el sitio Agua Amarga, en la finca homónima (distrito El Totoral, departamento de Tupungato), sobre el arroyo Agua Amarga, y entre la ruta provincial $\mathrm{n}^{\circ} 89 \mathrm{y}$ el Corredor Productivo (ruta provincial $n^{\circ}$ 52) (S33 $28^{\prime} 18.5^{\prime \prime}$ W69 11'29.3”) (Figura 1). El sector, ubicado en el piedemonte entre los 1.200 y $1.000 \mathrm{msm}$, es un glacis de acumulación cubierto por profundas capas de material aluvional, de pendiente aproximada entre $2^{\circ}$ y $10^{\circ}$ (Gutiérrez de Manchón y Furlani de Civit 1997:233).

Desde las primeras instalaciones españolas en el siglo XVII hasta mediados del siglo XX el área se destinó a la cría y engorde de ganado vacuno, mientras la planicie inmediata se transformó progresivamente mediante la agricultura bajo riego. Como consecuencia de cambios en las decisiones políticas y empresariales se produjo una transformación en la producción agrícola a partir de la década de 1990, incorporando la frontera pedemontana al sector bajo explotación frutivitivinícola (De Luca y Soria 1995; Gutiérrez de Manchón y Furlani de Civit 1997).

Las actividades agrícolas modernas comenzaron en la finca Agua Amarga en la década de 1970, con la instalación de 23,5 ha de parra sobre la margen derecha del arroyo. En la actualidad, la margen izquierda está destinada al cultivo de árboles frutales (membrillos, manzanos, duraznos, cerezos y ciruelos) y también se aprovechan algunos sectores bajos de la barranca del arroyo. El Parral y la Quinta ocupan una superficie aproximada de 52 ha, la cual coincide parcialmente con la de las concentraciones de material arqueológico.

\section{Dificultades para la Investigación Arqueológica en Áreas Aradas}

Los yacimientos arqueológicos reocupados se encuentran sometidos a la disturbación constante generada por las actividades humanas. Entre ellas, la labranza mecánica constituye uno de los procesos antrópicos que más afectan la disposición del registro arqueológico. En el Centro Oeste argentino la expansión de la explotación frutivitivinícola, con la incorporación de terrenos agrícolas óptimos, tiene como consecuencia la reocupación de sitios arqueológicos, como hemos observado en el Valle de Uco. Esta problemática ha sido destacada igualmente en sitios de los fértiles valles del centro de Chile (Hermosilla et al. 2003, 2005; Planella y Stehberg 1997).

Eludir el estudio de los "sitios arados" limita la posibilidad de conocer una parte del registro arqueológico y, desde la perspectiva de investigación regional y distribucional, introduce una desviación significativa en el procedimiento de muestreo, por lo que se han propuesto varias alternativas para trabajar en sectores sometidos a la labranza (Boismier 1997; Diez Martín 1998, 2003; Dunnell y Simek 1995; Lewarch y O'Brien 1981; Odell y Cowan 1987; entre otros).

Algunos estudios que se han realizado sobre "arqueología de zonas aradas" (ploughzone archaeology) sostienen que los efectos disturbadores de la agricultura son menos significativos de lo que comúnmente se cree (Lewarch y O'Brien 1981). Mediante la observación controlada de las actividades de labranza, la reproducción de las mismas en diseños experimentales y de simulación, concluyen que los efectos producidos por las labores agrícolas pueden ser: desplazamiento (vertical y horizontal) de los materiales, con la consecuente alteración de las agrupaciones y la estructura estratigráfica originales; cambios en la representatividad de las muestras superficiales en cuanto a la cantidad y clase en relación con los materiales enterrados, y cambios en las condiciones de preservación de los objetos.

Se ha demostrado que el mayor desplazamiento de los artefactos se produce en la dirección de la labranza y que el desplazamiento lateral no es significativo (Odell y Cowan 1987; Roper 1976). Lewarch y O'Brien (1981), entre otros, sostienen que la alternancia temporal de la orientación de la labranza introduce correcciones y un equilibrio 


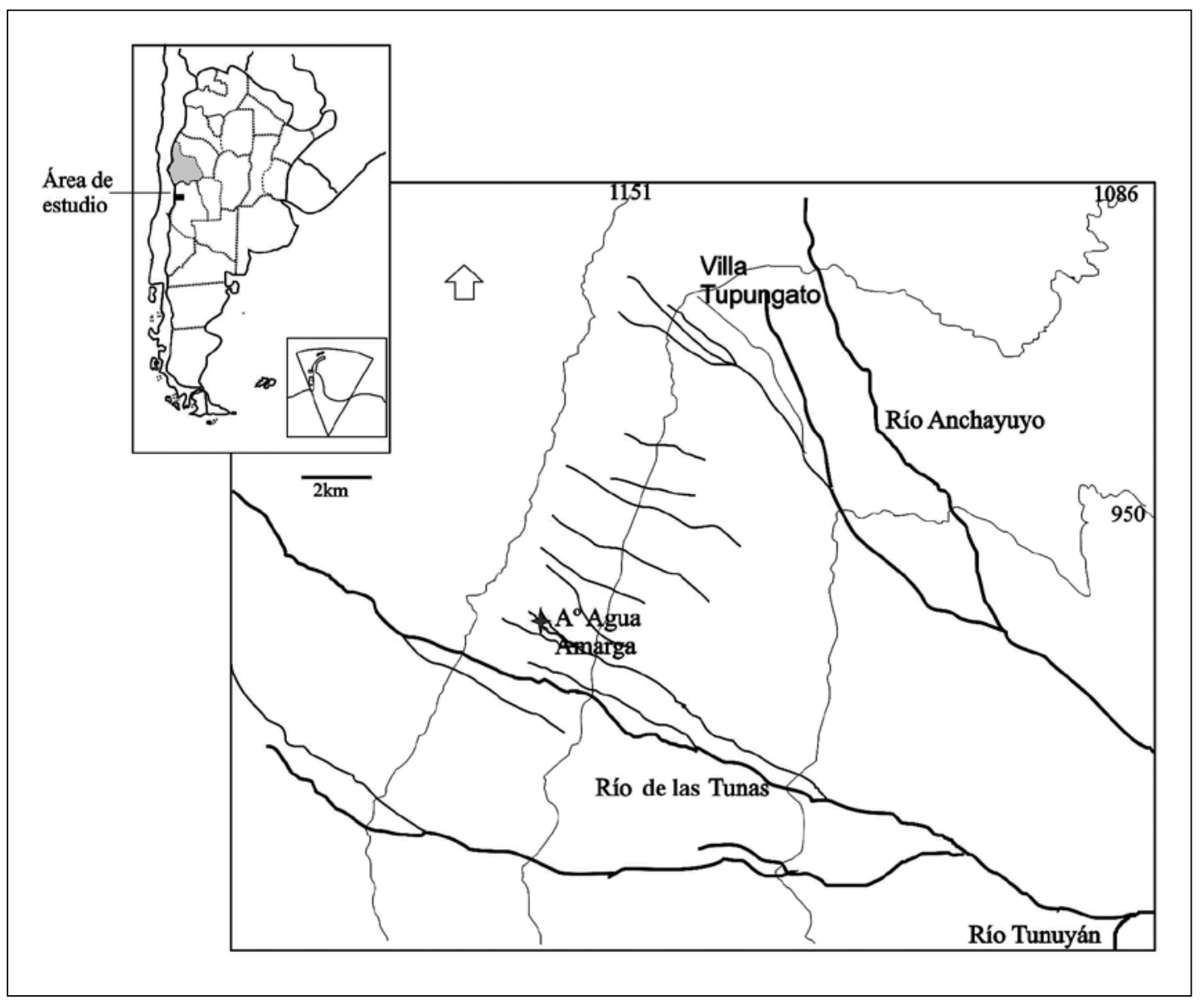

Figura 1. Ubicación del sitio Agua Amarga. Modificado de la Carta Topográfica de la República Argentina, Instituto Geográfico Militar, 3369-27. Mendoza, 1927.

Location of Agua Amarga site. Map based on the Topographic Chart of República Argentina, Instituto Geográfico Militar, 3369 27. Mendoza, 1927.

en la distribución de los materiales, pero trabajos posteriores han disentido con esta afirmación sobre la estabilidad de los patrones de dispersión de los artefactos, ya que no parece existir empíricamente (Boismier 1997:237).

El llamado "efecto dimensional" (size effect, Baker 1978) se refiere a la distorsión aparente en las colecciones superficiales por la cual los artefactos más grandes tienen una representación mayor en la superficie en relación con su proporción en la población total. Sin embargo, las alteraciones en la representatividad de los artefactos por tamaño ha sido objeto de discusiones. En tanto que los distintos componentes ("subpoblaciones") están representados proporcionalmente en la superficie: los tipos más numerosos están mejor representados, mientras que los menos abundantes están representados en la superficie en una proporción muy baja (Boismier 1997:234).

A partir del control de grupos experimentales, Boismier (1997) propone una serie de criterios útiles para la identificación del grado de modificación producido por la labranza en sitios arqueológicos en tres niveles: inicial, intermedio o avanzado y final o terminal. El reconocimiento de patrones se relaciona con la evaluación de los efectos de la labranza en las características de tres tipos de restos: el artefacto, la muestra total de artefactos y el agrupamiento de artefactos. Por un lado, se analizan los rasgos dejados por la labranza en los artefactos individuales, mientras que los patrones de composición de los conjuntos y la configuración 
espacial de la muestra total de artefactos son usados para juzgar el estado relativo de los cambios inducidos por la labranza en las distribuciones de artefactos y para identificar cualquier distribución que pueda reflejar la estructura intrasitio.

Entre las transformaciones en la configuración espacial se destacan el incremento del área y la consecuente reducción de la densidad de artefactos y la uniformidad o "amalgama" de los agrupamientos intrasitio (Boismier 1997:235). Las agrupaciones de artefactos adquieren una forma menos distintiva y más circular, uniéndose los agrupamientos espacialmente adyacentes a medida que se suceden las operaciones en grupos que continuamente serán más grandes. Los límites serán cada vez más difusos y el sitio adquirirá la orientación de la pendiente y asociaciones topográficas.

Respecto de la conservación de los artefactos, se observa que la labranza produce fracturas y la desintegración de los más frágiles. Desde el inicio de estas actividades se observan frecuencias altas de materiales de tamaño grande y mediano en la composición del conjunto, pero al incrementarse estas operaciones, aumenta la frecuencia de bordes dañados y artefactos rotos y disminuye la densidad superficial. Como consecuencia, se incrementan las frecuencias de artefactos de pequeño tamaño en la muestra superficial, alterando la composición de los conjuntos ya que los elementos más durables tendrán una representación incrementada (Boismier 1997:238). El volumen, la forma y el tamaño de los objetos influyen en su conservación y transporte por distintos procesos erosionales.

En general, los estudios comentados han observado y controlado el efecto de la labranza mecánica, desatendiendo otros procesos antrópicos modificadores del registro arqueológico, como pueden ser el riego; el pisoteo consecuencia del riego, la cosecha, el atado y la poda de la parra; la recolección selectiva, entre otros. Por ejemplo, el efecto del pisoteo en la formación de los sitios arqueológicos ha sido objeto de estudios experimentales que identificaron alteraciones en las asociaciones estratigráficas, en las distribuciones horizontales y la conservación de los artefactos (Civalero de Biset 1991; Nielsen 1991; entre otros), datos que podrían considerarse al analizar estos procesos.

\section{Agricultura en Agua Amarga}

Las conclusiones comentadas son resultado de situaciones experimentales y simulaciones, sin embargo los procedimientos varían de acuerdo al tipo de suelo y/o de cultivos. En la finca Agua Amarga, las operaciones iniciales de nivelación y preparación del terreno provocaron transformaciones mediante el desplazamiento de grandes volúmenes de sedimentos desde zonas elevadas para el relleno de otras deprimidas y el uso de arado de discos, niveleta y subsolador para reducir la dureza del suelo y desprender los residuos y otros agregados. El arado de discos tiene una profundidad de labor entre $0,30 \mathrm{y}$ $0,50 \mathrm{~cm}$, permitiendo una roturación suave del suelo, sobre todo para eliminar malezas superficiales sin provocar pérdidas de materia orgánica ni romper la estructura del suelo, facilitando su erosión por el viento. Los subsoladores y arados de subsuelo que se utilizan para prevenir la erosión, rompen y pulverizan el suelo sin invertirlo (Hunt 1983:112).

Actualmente, el sector de la finca ocupado por el parral (23,5 ha) se encuentra sometido a menor impacto de la maquinaria agrícola ("labranza reducida o mínima", Studdert 2001) y riego ${ }^{3}$. El tractor se utiliza para desmalezar, cortando la hierba al ras, pero sin arrancarla ni incorporarla al suelo; y para suministrar pesticidas, siguiendo la dirección $\mathrm{N}-\mathrm{S}$ de las hileras de parra, según el modelo de alternación traslapada (Hunt 1983:20). El sistema de riego por goteo permite optimizar el consumo de agua, adecuándola al valor apropiado para el cultivo bajo condiciones determinadas de suelo y clima, e incorpora fertilizantes sin necesidad de roturar ni voltear el suelo.

El sector ocupado por la quinta de frutales está sometido a un mayor impacto por el arado de disco para eliminar malezas y mover la tierra, al menos una vez al año y por el sistema de riego por "melga" o "manto" (en el parral se aplica este sistema en épocas en que abunda el recurso hídrico). El riego por manto consiste en la inundación de toda la superficie cultivada, para lo cual es necesario intervenir con una herramienta (zapa) para obstruir o facilitar el paso del agua.

Una franja de tamaño variable no mayor a $10 \mathrm{~m}$ desde la barranca del cauce se conservaba sin cultivar; sin embargo observamos otros procesos que han incidido en la margen derecha, como el tránsito de vehículos, maquinaria para remover terreno, desmalezar y otras actividades. Asimismo, el sedimento se presenta muy suelto y homogéneo 
debido a la acción de roedores (Ctenomis mendocinus), cuyas cuevas se observan en las paredes de la barranca y fueron identificadas también en la excavación, entre otros restos (huesos, deyecciones, nidos). Estos procesos no se identifican en la margen izquierda que, en cambio, conserva vegetación de monte.

\section{Metodología}

Las condiciones de visibilidad del sitio impiden identificar la superficie de distribución de los artefactos en la finca. Por razones operativas, al desconocer los límites de la distribución y por tanto el tamaño de la población, decidimos superponer el diseño del muestreo al del cultivo de la finca, y utilizamos como unidad de muestreo la transecta formada por el espacio entre dos hileras consecutivas de parra y frutales en los respectivos sectores. Realizamos un muestreo probabilístico de tipo sistemático (Shennan 1992:295, 313-316) del 5\% del total de transectas (920), muestreando una cada 20 a partir de una seleccionada al azar ${ }^{4}$. El sector de Parral cuenta con 553 transectas y el de Quinta con 367. Dentro de cada una, los parrales están sembrados a $3 \mathrm{~m}$ de distancia, conformando cuadrículas de $9 \mathrm{~m}^{2}$ (dos plantas consecutivas y dos enfrentadas) y los frutales delimitan cuadrículas de aproximadamente $11 \mathrm{o}$ $12 \mathrm{~m}^{2}$ (duraznos y ciruelos $4 \times 3 \mathrm{~m}$; cerezos $4,7 \times 2,4$ m; manzanos 4,8 x 2,3 m). Las cuadrículas (1.491 en total) fueron utilizadas como subdivisiones de las transectas para realizar el muestreo y las operaciones estadísticas (Figuras 2, 3 y 4). La selección del tamaño y la forma de la cuadrícula se realizó con un criterio operativo, aprovechando un espacio delimitado que constituye una unidad de trabajo agrícola.

El tipo de distribución de los materiales en el espacio se analizó mediante la Prueba de razón varianza/media $\left(\mathrm{S}^{2} / \mathrm{x}\right)$. Ésta consiste en un método de cuadrados de análisis de distribución de puntos y está basada en una propiedad de la distribución de Poisson según la cual su media y su varianza son iguales. La razón $\mathrm{S}^{2} / \mathrm{x}$ será igual a 1 para una distribución de puntos aleatoria; menor a 1 para una distribución regular y mayor a 1 para distribuciones agrupadas. La significación de las desviaciones de la razón observada se verifica a partir del valor de uno comparando el índice de dispersión $\left(\mathrm{S}^{2} / \mathrm{x}\right) \mathrm{x}$ (n-1), donde $\mathrm{n}$ es el número de cuadrados, con la estadística de $\mathrm{x}^{2}$ para $\mathrm{n}-1$ grados de libertad (Hodder y Orton 1990:45).

El muestreo superficial se complementó con la excavación de 13 sondeos de $1 \mathrm{~m}^{2}$, uno de los cuales se amplió con otras 8 cuadrículas de $1 \mathrm{~m}^{2}$. La ubicación de los sondeos estuvo restringida a los sectores donde nos fue permitido excavar, principalmente en las proximidades de la barranca del cauce, espacio no cultivado y por tanto poco impactado por la labranza (Sondeos I, II, IV y XIII sobre la margen sur del arroyo, y VI, VIII, IX, X, XI y XII sobre la margen norte, ubicación en la Figura 2). Asimismo, pudimos excavar algunos sondeos dentro del área cultivada en el sector de Parral (Sondeos III, V y VII) (Figura 2).

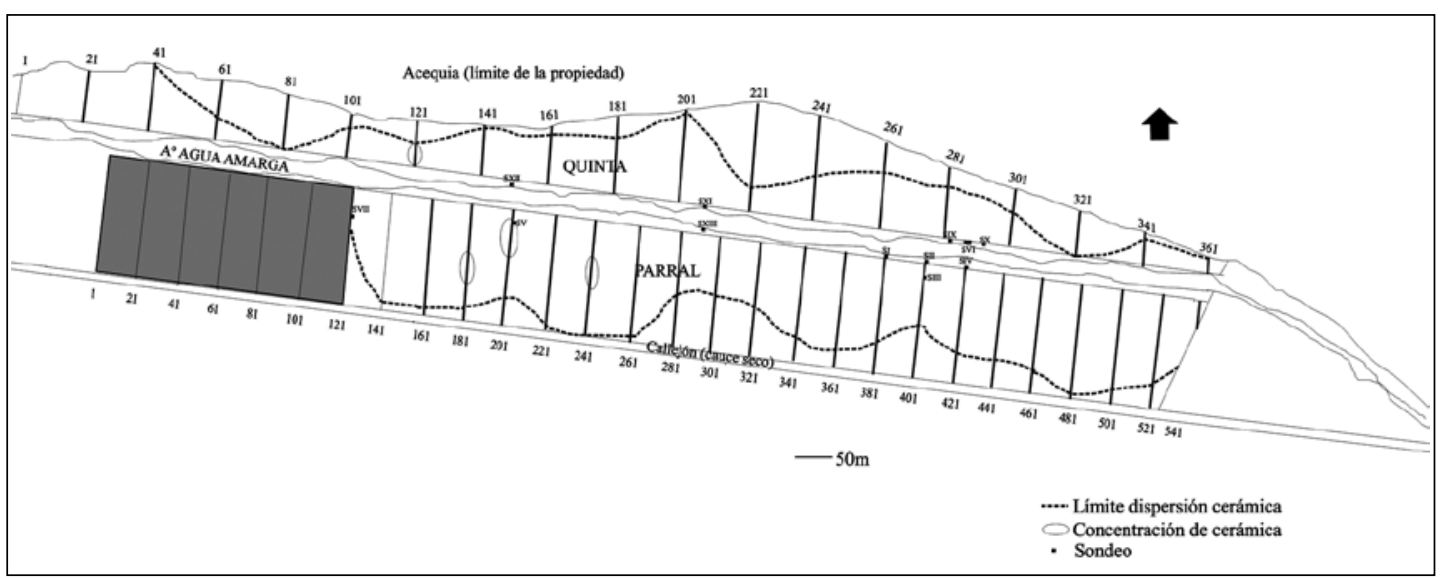

Figura 2. Agua Amarga: ubicación de transectas, sondeos, límites de la dispersión y sectores de concentración de la cerámica. Modificado del Plano de Agua Amarga, Dirección Provincial de Catastro, julio de 1988.

Agua Amarga: Location of transects, test pits, and limits of distribution and sectors of concentration of potsherd. Map based on Plan Map of Agua Amarga, Dirección Provincial de Catastro, July 1988. 
hilera

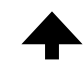

$\begin{array}{lllllllllllllllllllllllllllllll}1 & 21 & 41 & 61 & 81 & 101 & 121 & 141 & 161 & 181 & 201 & 221 & 241 & 261 & 281 & 301 & 321 & 341 & 361 & 381 & 401 & 421 & 441 & 461 & 481 & 501 & 521 & 541 & 561 & \text { Cuad }\end{array}$

\begin{tabular}{|l|l|l|l|l|l|l|l|l|l|l|l|l|l|l|l|l|l|l|l|l|l|l|l|l|l|l|l|l|l|l|}
\hline & & & & & & 0 & 0 & & 0 & 0,2 & & 0,4 & 0,4 & 0,3 & 0 & 0,1 & 0 & 0 & 0 & 0 & 0,7 & 0 & 0 & 0 & 0 & 0 & 0 & 0 \\
\hline
\end{tabular}

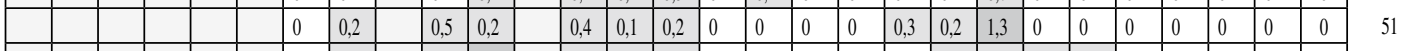

\begin{tabular}{|l|l|l|l|l|l|l|l|l|l|l|l|l|l|l|l|l|l|l|l|l|l|l|l|l|l|l|l|l|}
\hline & & & & & 0 & 0,4 & & 0,6 & 1,3 & & 0,2 & 0,5 & 0,1 & 0 & 0 & 0 & 0 & 0 & 0,6 & 0,4 & 0,1 & 0,1 & 0 & 0 & 0 & 0 & 0 \\
\hline & & & & & 0,1 & 0 & & 0,5 & 1,4 & & 0 & 0,4 & 0 & 0 & 0 & 0 & 0,2 & 0,2 & 0,6 & 0,4 & 0,4 & 0 & 0 & 0 & 0 & 0 & 0 \\
\hline
\end{tabular}

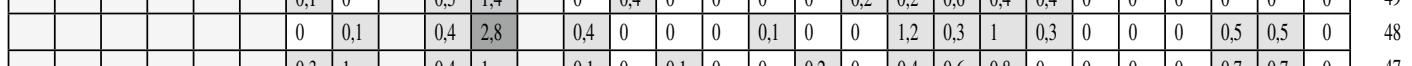

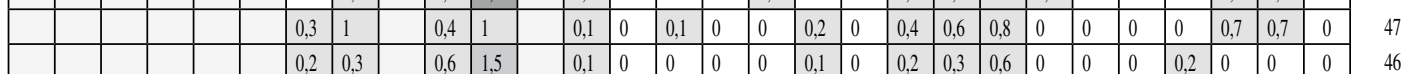

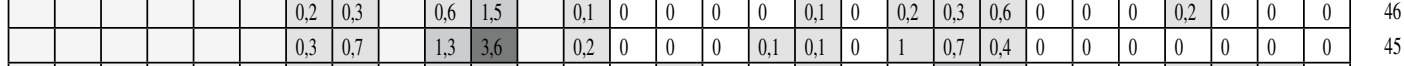

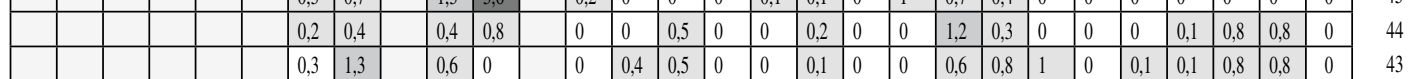

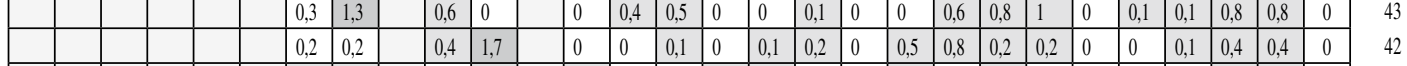

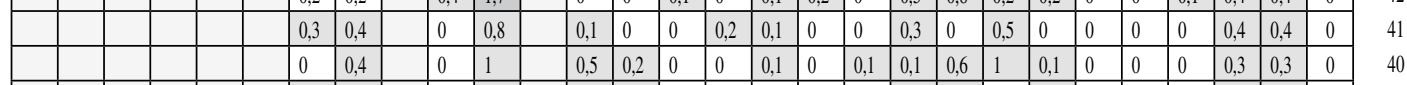

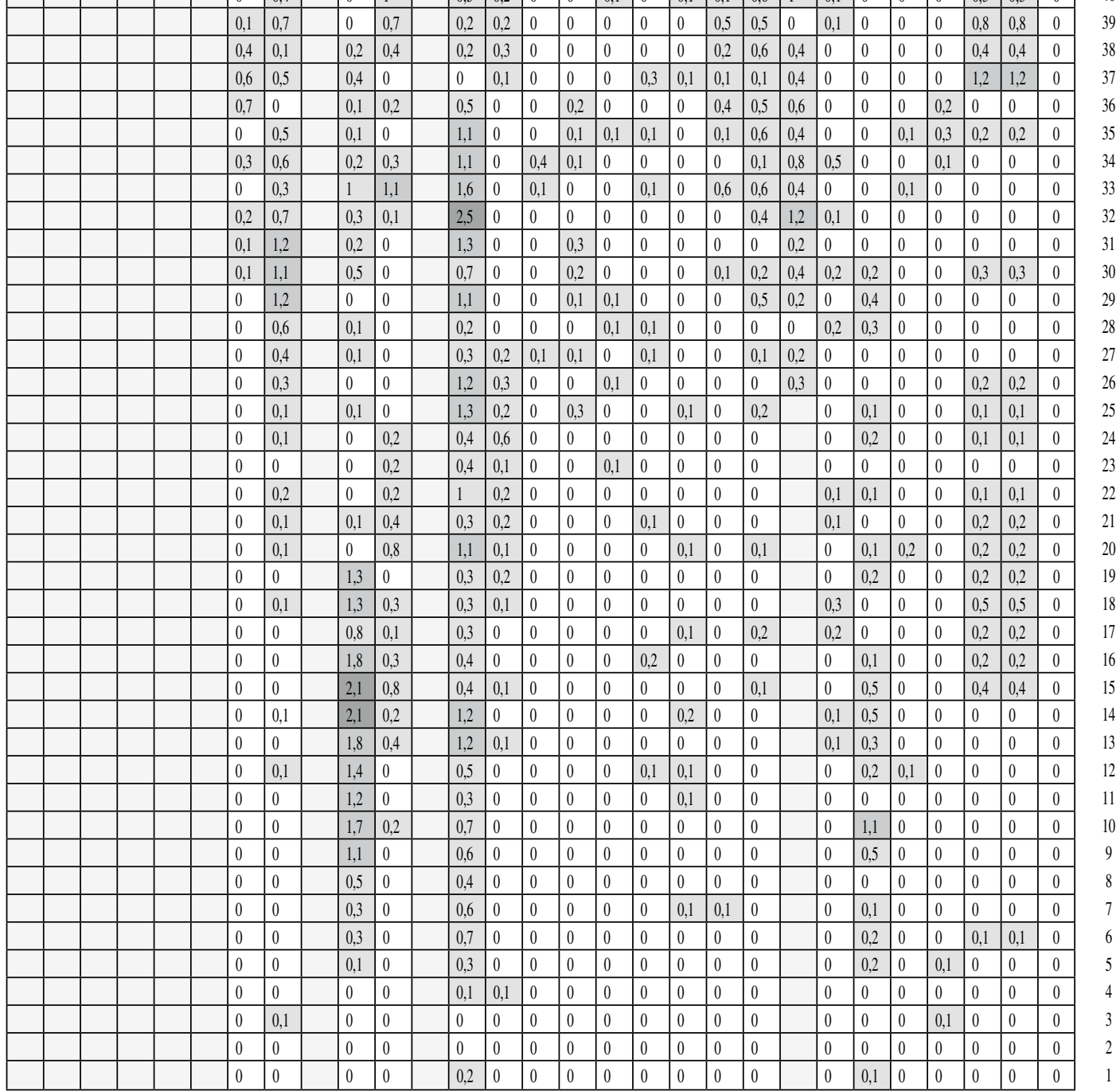

\begin{tabular}{|l|l|l}
0 & \\
$0,01 / 1$ & $1,1 / 2$ \\
$2,1 / 3$
\end{tabular}$\square \begin{aligned} & 3,1 / 4 \\
& \text { Sector inundado }\end{aligned}$

Figura 3. Esquema de distribución de la cerámica en Agua Amarga: densidad de fragmentos por m² en el Parral. Representación de las transectas de recolección.

Schematic pottery distribution at Agua Amarga: density of potsherds per $m^{2}$ (Parral). Taken from surface collections by transects. 
$1 \quad 2141 \quad 6181101121141161181201221241261281301321341361$ cuad

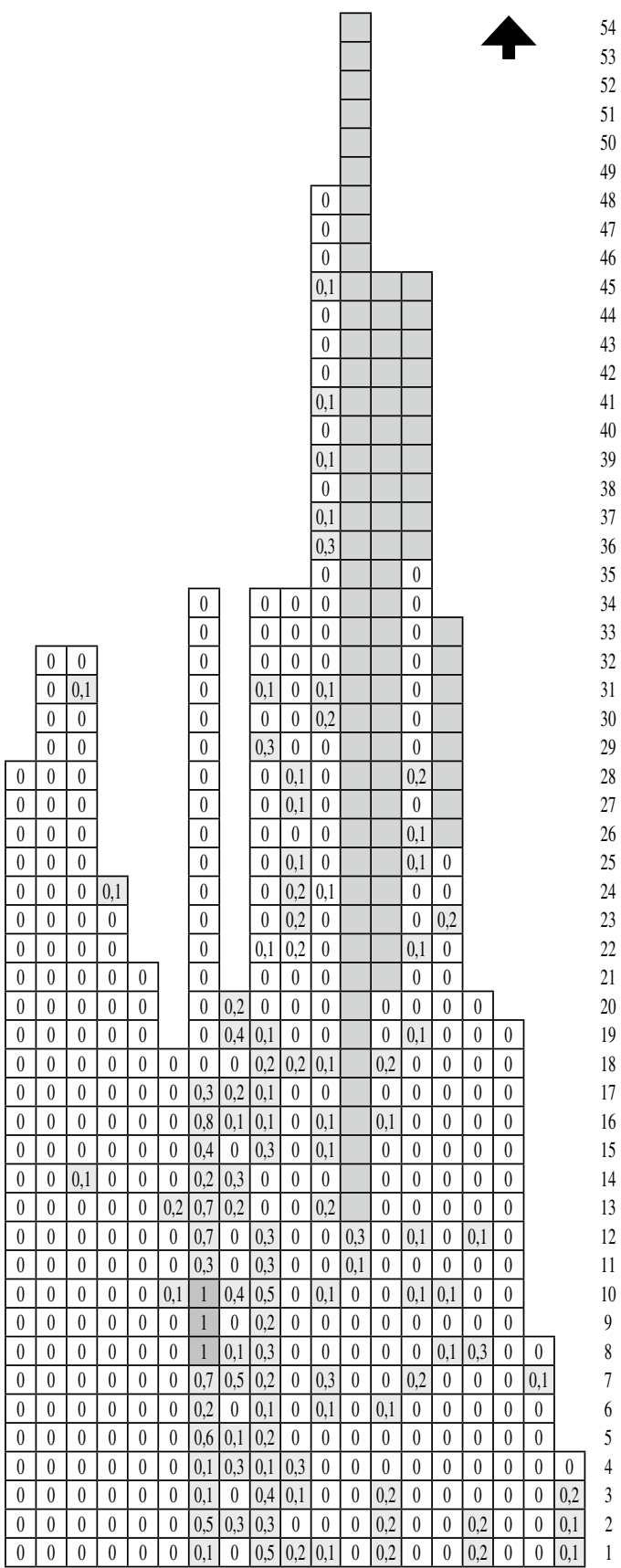

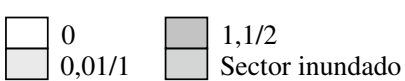

Figura 4. Esquema de distribución de la cerámica en Agua Amarga: densidad de fragmentos por $\mathrm{m}^{2}$ en la Quinta. Representación de las transectas de recolección.

Schematic pottery distribution at Agua Amarga: density of potsherds per $m^{2}$ (Quinta). Taken from surface collections by transects.
Debido a la homogeneidad de la matriz sedimentaria, la excavación se realizó mediante niveles de $10 \mathrm{~cm}$, registrando la profundidad de las ocupaciones que pudieran identificarse. Seguimos la propuesta metodológica utilizada por Steinberg y Diez Martín (2003), entre otros, para establecer las características del efecto dimensional en relación con el movimiento vertical. El modelo toma como unidad de análisis al sondeo de $1 \mathrm{~m}^{2}$ y considera a los sedimentos como un flujo "teniendo en cuenta que un $\mathrm{m}^{3}$ es equiparable a 1.000 litros de sedimento, el fluido será estimado por litros de tierra..." (Diez Martín 2003:49). Según estas experiencias, una muestra de 100 litros de sedimento es suficiente para obtener una representación de la densidad de objetos presentes en el subsuelo.

La comparación entre las muestras de superficie y de excavación se estableció mediante pruebas estadísticas (R de Pearson y t de Student) y se ilustró mediante tablas y gráficos de frecuencias 5 .

Para identificar la representatividad de la cerámica de superficie se consideró la frecuencia y el peso de los tiestos. La selección de esta última variable en lugar de otra, dimensional, se recomienda por ser más rápida de calcular para trabajar con una muestra grande (Dunnell y Simek 1995; Orton et al. 1997[1993]). Dunnell y Simek (1995) han demostrado que la resistencia de los tiestos a la fractura está determinada por su espesor, y suponen una relación entre el peso de la cerámica y su espesor, cualquiera sea el tipo de pasta. A pesar de la variación tecnológica de los tipos comparados, el promedio de espesor es similar (alrededor de $0,6 \mathrm{~cm}$ ), por lo que suponemos que el peso es una variable adecuada para los propósitos de este trabajo.

La clasificación estilística de la cerámica se realizó mediante el análisis tecnotipológico de los tiestos, de acuerdo a los procedimientos descriptivos sugeridos por Orton et al. (1997[1993]).

\section{Resultados}

Del muestreo superficial se obtuvo un total de 2.368 restos arqueológicos, de los cuales el $80 \%$ es cerámica -1.845 fragmentos-. La ubicación de las transectas se grafica en la Figura 2, en tanto que los esquemas de las Figuras 3 y 4 simplifican la representación de la distribución por $\mathrm{m}^{2}$ de la cerámica en los sectores de Parral -margen derecha-y Quinta -margen izquierda-respectivamente, ya que las transectas están presentadas en forma 
continua. Observamos que hay mayor cantidad de cerámica en el primer sector $(0,18$ fragmentos por $\mathrm{m}^{2}$ ) y que las mayores densidades se concentran en las hileras 181 y 201. Los tiestos tienen una distribución de tipo agrupada de acuerdo con la prueba de razón $S^{2} / x$ (Tabla 1). En el sector de Quinta la frecuencia de cerámica en superficie es muy baja $\left(0,05\right.$ fragmentos por $\left.\mathrm{m}^{2}\right)$, concentrándose en las cuadrículas de la hilera 81 más cercanas al cauce, con una distribución también agrupada.

Si tenemos en cuenta que el sitio presenta una acentuada pendiente con dirección noroeste-sureste (ver cotas en la Figura 1) y que el laboreo se realiza siguiendo una dirección norte-sur, se puede observar una relación con la distribución de los materiales en la superficie. La forma del sitio coincide, en gran parte, con la de la finca (Figura 2). No se identificó el tamaño o la forma de las concentraciones dentro del sitio, sino solamente los lugares de mayor densidad de materiales. Podríamos acordar con otros trabajos comentados que las operaciones de labranza producen la unión de las agrupaciones, sin embargo el tipo de muestreo nos limita para establecer este tipo de conclusiones.

En el sector de Parral el sedimento arcilloso se presenta muy duro y compacto en los niveles iniciales (aproximadamente hasta $40 \mathrm{~cm}$ ), con raíces. En estos niveles no se identifican contextos ni integridad en el registro arqueológico. En cambio, reconocimos dos contextos en los espacios sin cultivar en las proximidades de las barrancas sur y norte respectivamente (a 20 y $27 \mathrm{~cm}$ de profundidad, respectivamente), donde no se observan alteraciones en la conservación e integridad del registro (Ots 2007:184-185).

Tabla 1. Análisis de la distribución de la cerámica. Resultado de la prueba de razón varianza/media. Analysis of pottery sherds distribution. Variance/mean ratio test.

\begin{tabular}{cc}
\hline Parral & Quinta \\
\hline $\mathrm{x}=1,63$ & $\mathrm{x}=0,59$ \\
$\mathrm{~S}^{2}=10,7$ & $\mathrm{~S}^{2}=2,51$ \\
$\mathrm{~S}^{2} / \mathrm{x}=6,57$ & $\mathrm{~S}^{2} / \mathrm{x}=4,28$ \\
$\mathrm{P}$ valor $<10^{-15}$ & $\mathrm{P}$ valor $<10^{-10}$ \\
\hline
\end{tabular}

Ho: "La distribución del número de fragmentos por cuadrícula es Poisson".

Hi: "La distribución del número de fragmentos por cuadrícula no es Poisson".

Rechazo Ho $\leftrightarrow$ p valor $<\alpha$.
De las excavaciones se obtuvieron 1.145 fragmentos de cerámica, además de material lítico, óseo, carbón, granos de Zea mays, Phaseolus spp. y semillas de Cucurbita spp. termoalteradas, cáscara de huevo de Rhea americana (natural y quemada), arcilla cocida. Si bien el análisis de estos materiales y sus asociaciones no se incluyen en este trabajo, podemos adelantar que corresponderían al menos a dos ocupaciones del período agroalfarero temprano-medio y tardío, respectivamente, a partir del registro cerámico y de acuerdo al proceso cultural regional.

El 9,79\% de los fragmentos de cerámica recolectados en la superficie y en los sondeos (295 tiestos) están decorados. En la Tabla 2 mostramos la cantidad de fragmentos (n) y la proporción sobre el total (\%), agrupados de acuerdo al acabado de superficie (alisado, con o sin decoración) y al color de las pastas. Asimismo, se consideró el peso $(7.251 \mathrm{~g}$ en total, $\mathrm{x}=2,43 \mathrm{~g}$ ) (Tabla 3).

Se analizaron las pastas y los patrones formales y decorativos que permitieron la identificación de los tres componentes típicos de la etapa agroalfarera regional:

(1) Cerámica gris, marrón grisácea o marrón rojiza alisada y con decoración geométrica incisa con motivos de líneas finas paralelas o líneas quebradas angularmente y paralelas -“chevrones"-, o incisiones en forma de puntos alargados. Recipientes con esta decoración fueron adscriptos al agroalfarero temprano y medio del norte y centro

Tabla 2. Distribución de la cerámica de Agua Amarga según color de pasta y tratamiento superficial.

Pottery sherds distribution from Agua Amarga by color and superficial treatment.

\begin{tabular}{llrl}
\hline Grupo & \multicolumn{1}{c}{ Subgrupo } & $\mathrm{n}$ & $\%$ \\
\hline \multirow{4}{*}{ Decorado } & Gris inciso & 14 & 0,47 \\
& Marrón inciso & 15 & 0,5 \\
& Rojo-negro sobre naranja & 253 & 8,46 \\
& Rojo-negro sobre blanco & 13 & 0,43 \\
\hline & Total & 295 & 9,87 \\
\hline \multirow{5}{*}{ Sin decorar } & Gris & 1.210 & 40,5 \\
& Narrón & 1.183 & 39,57 \\
& Rojo & 268 & 8,96 \\
& Indeterminado & 20 & 0,67 \\
& Total & 14 & 0,47 \\
\hline \multirow{2}{*}{ Total } & & 2.695 & 90,13 \\
\hline
\end{tabular}


Tabla 3. Frecuencia de pesos de la cerámica de Agua Amarga según tratamiento superficial. Frequency of pottery weight from Agua Amarga by superficial treatment.

\begin{tabular}{lrrrrr}
\hline Tratamiento superficie & $\mathrm{n}$ & $\%$ & Peso $(\mathrm{g})$ & $\%$ & Relación peso/fragm $(\mathrm{g})$ \\
\hline Decorada & 295 & 9,9 & 643,87 & 8,9 & 2,18 \\
Sin decorar & 2.695 & 90,1 & $6.607,13$ & 91,1 & 2,45 \\
\hline Total & 2.990 & 100 & 7.251 & 100 & \\
\hline
\end{tabular}

Tabla 4. Promedio de peso de la cerámica de Agua Amarga por profundidad. Pottery sherds weight mean from Agua Amarga by depth.

\begin{tabular}{|c|c|c|c|c|c|c|c|c|c|c|c|c|c|c|c|c|c|c|c|}
\hline $\begin{array}{l}\text { Prof. } \\
(\mathrm{cm})\end{array}$ & $\begin{array}{r}0 \\
10\end{array}$ & $\begin{array}{l}10 \\
20\end{array}$ & $\begin{array}{l}20 \\
30\end{array}$ & $\begin{array}{l}30 \\
40\end{array}$ & $\begin{array}{l}40 \\
50\end{array}$ & $\begin{array}{l}50 \\
60\end{array}$ & $\begin{array}{l}60 \\
70\end{array}$ & $\begin{array}{l}70 \\
80\end{array}$ & $\begin{array}{l}80 \\
90\end{array}$ & $\begin{array}{r}90 \\
100\end{array}$ & $\begin{array}{l}100 \\
110\end{array}$ & $\begin{array}{l}110 \\
120\end{array}$ & $\begin{array}{l}120 \\
130\end{array}$ & $\begin{array}{l}130 \\
140\end{array}$ & $\begin{array}{l}140 \\
150\end{array}$ & $\begin{array}{l}150 \\
160\end{array}$ & $\begin{array}{l}160 \\
170\end{array}$ & $\begin{array}{l}170 \\
180\end{array}$ & $\begin{array}{l}180 \\
190\end{array}$ \\
\hline $\mathrm{n}$ & 368 & 203 & 181 & 93 & 43 & 108 & 49 & 11 & 18 & 8 & 11 & 14 & 11 & 7 & 13 & 8 & 2 & 6 & 1 \\
\hline $\begin{array}{l}\text { Peso total } \\
\quad(\mathrm{g})\end{array}$ & 632 & 476 & 638 & 270 & 136 & 393 & 126 & 33,3 & 38,4 & 11,6 & 34,6 & 52,2 & 21,3 & 26,1 & 33,3 & 16,1 & 3,61 & 18,7 & 1,54 \\
\hline $\mathrm{X}$ & 1,7 & 2,3 & 3,5 & 2,9 & 3,2 & 3,6 & 2,6 & 3,02 & 2,1 & 1,45 & 3,1 & 3,7 & 1,9 & 3,7 & 2,6 & 2,01 & 1,8 & 3,1 & \\
\hline$S$ & 2,5 & 3,4 & 4,9 & 3,9 & 4,1 & 5,04 & 2,8 & 2,4 & 2,3 & 1,3 & 3,6 & 4,85 & 1,2 & 6,9 & 1,95 & 1,9 & 0,5 & 3,6 & \\
\hline
\end{tabular}

de Mendoza (siglos VI-XI) (Bárcena 1998, 2001; Canals Frau 1956; Canals Frau y Semper 1956). Los fragmentos analizados corresponden al cuello y/o cuerpo de grandes vasijas con cuerpo globular, cuello recto y alto y boca ancha y tienen un espesor entre 0,7 y $0,9 \mathrm{~cm}$.

Algunos fragmentos del tipo mencionado se encontraron en la superficie del sitio, en tanto que en los Sondeos VI, VIII, IX y X se descubrieron entre 27 y $35 \mathrm{~cm}$ de profundidad, en asociación con otros fragmentos de color gris y marrón grisáceo alisados y pulidos, sin decoración, y con artefactos líticos (lascas y manos de moler fragmentadas).

(2) Tiestos naranjas alisados y pulidos decorados con pintura geométrica negra y roja asociados a dos tipos que se han reconocido en el área para el agroalfarero tardío: por su forma y decoración, algunos fragmentos corresponden a las ollas características de la denominada cultura de Viluco, que se encuentran en varios sitios del Centro Oeste (Bárcena 2001; Cahiza 2003; Lagiglia 1978; Michieli 1998; Rusconi 1962; entre otros), coincidiendo dicha distribución con el área de ocupación huarpe. Se reconocieron atributos formales y decorativos de los pucos o escudillas típicos de la denominada facie II de Viluco o Viluco Inca (Lagiglia 1978:244).

Sin embargo, la mayoría de los fragmentos (de aproximadamente 0,6 ó $0,7 \mathrm{~cm}$ de espesor) son semejantes al tipo "rojizo o anaranjado pulido, pintado de rojo" del Tambo incaico de Tambillos, cuya descripción puede asimilarse a la de los aríbalos o aribaloides de este sitio (Bárcena y Román 1990 [1986-87]:41) $)^{6}$.

(3) Cerámica decorada muy fragmentada y en algunos casos no muy bien conservada, del tipo Diaguita Chileno Fase III o Inca. Los ejemplares recuperados son del tipo rojo engobado (ambas caras), con o sin decoración geométrica pintada en blanco y/o negro, y negro y/o rojo sobre engobe blanco.

Los tiestos Viluco y Diaguita Chileno Inca se registraron en la superficie y en los primeros niveles de excavación (hasta $30 \mathrm{~cm}$ ). Excepcionalmente, algunos fragmentos Viluco se encontraron también en niveles inferiores.

Hemos considerado la cantidad y el peso de la cerámica por niveles artificiales de aproximadamente $10 \mathrm{~cm}$ (100 litros de sedimento) en los Sondeos I a XIII (las 8 cuadrículas excavadas en extensión no ofrecen resultados comparables, porque no se excavaron con el mismo criterio). En la Tabla 4 presentamos el promedio (x) de peso general por nivel y la desviación standard (S) y en la Tabla 5 la frecuencia y promedio de peso por nivel por sondeo (en cada sondeo se indican los niveles finales de excavación, que corresponden a niveles de sedimento estéril). Los 1.850 tiestos recolectados de la superficie representan una densidad de 0,1 fragmento 
Tabla 5. Promedio de peso de la cerámica por niveles de $0,10 \mathrm{~m}$ por Sondeo.

Pottery sherds weight mean by $10 \mathrm{~cm}$ levels in I-XIII test pits.

\begin{tabular}{|c|c|c|c|c|c|c|c|c|c|c|c|c|c|}
\hline Prof. (cm) & SI & SII & SIII & SIV & SV & SVI & SVII & SVIII & SIX & sX & SXI & SXII & SXIII \\
\hline $0-10$ & $\begin{array}{c}2,4 \\
(12)\end{array}$ & $\begin{array}{c}1,4 \\
(12)\end{array}$ & $\begin{array}{l}1,1 \\
(43)\end{array}$ & $\begin{array}{c}3,8 \\
(11)\end{array}$ & $\begin{array}{c}1,2 \\
(88)\end{array}$ & $\begin{array}{c}1 \\
(1)\end{array}$ & $\begin{array}{c}1,9 \\
(16)\end{array}$ & $\begin{array}{l}1,9 \\
(6)\end{array}$ & $\begin{array}{l}0,7 \\
(2)\end{array}$ & $\begin{array}{l}1,8 \\
(8)\end{array}$ & $\begin{array}{l}0,9 \\
(7)\end{array}$ & $\begin{array}{c}1,3 \\
(64)\end{array}$ & $\begin{array}{c}3,5 \\
(13)\end{array}$ \\
\hline $10-20$ & $\begin{array}{c}4,9 \\
(11)\end{array}$ & $\begin{array}{c}3,4 \\
(12)\end{array}$ & $\begin{array}{l}1,7 \\
(33)\end{array}$ & $\begin{array}{l}2,9 \\
(2)\end{array}$ & $\begin{array}{l}2,3 \\
(46)\end{array}$ & $\begin{array}{l}1,4 \\
(6)\end{array}$ & $\begin{array}{l}1,5 \\
(9)\end{array}$ & $\begin{array}{l}2,1 \\
(8)\end{array}$ & $\begin{array}{l}2,6 \\
(8)\end{array}$ & $\begin{array}{l}2,4 \\
(15)\end{array}$ & $\begin{array}{l}8,8 \\
(4)\end{array}$ & $\begin{array}{c}1,5 \\
(40)\end{array}$ & $\begin{array}{l}2,4 \\
(6)\end{array}$ \\
\hline $20-30$ & $\begin{array}{c}2 \\
(2)\end{array}$ & $\begin{array}{c}2,8 \\
(13)\end{array}$ & $\begin{array}{c}3,2 \\
(50)\end{array}$ & $\begin{array}{l}3,2 \\
(2)\end{array}$ & $\begin{array}{l}4,1 \\
(20)\end{array}$ & $\begin{array}{l}6,4 \\
(3)\end{array}$ & $\begin{array}{l}1,8 \\
(2)\end{array}$ & $\begin{array}{l}0,7 \\
(2)\end{array}$ & - & $\begin{array}{c}3 \\
(19)\end{array}$ & $\begin{array}{l}0,8 \\
(3)\end{array}$ & $\begin{array}{c}2,9 \\
(33)\end{array}$ & $\begin{array}{l}3,7 \\
(8)\end{array}$ \\
\hline $30-40$ & - & $\begin{array}{c}3,9 \\
(13)\end{array}$ & $\begin{array}{l}3,5 \\
(23)\end{array}$ & $\begin{array}{l}4,9 \\
(1)\end{array}$ & $\begin{array}{l}2,2 \\
\text { (13) }\end{array}$ & $\begin{array}{l}0,6 \\
(5)\end{array}$ & - & $\begin{array}{l}1,1 \\
(6)\end{array}$ & $\begin{array}{l}3,3 \\
(3)\end{array}$ & $\begin{array}{l}4,1 \\
\text { (11) }\end{array}$ & $\begin{array}{l}1,7 \\
(10)\end{array}$ & $\begin{array}{l}1,8 \\
(2)\end{array}$ & $\begin{array}{l}1,1 \\
(2)\end{array}$ \\
\hline $40-50$ & - & $\begin{array}{c}7,6 \\
(6)\end{array}$ & $\begin{array}{l}2,5 \\
(3)\end{array}$ & $\begin{array}{l}1,8 \\
(1)\end{array}$ & - & $\begin{array}{l}6,5 \\
(2)\end{array}$ & - & $\begin{array}{l}2,3 \\
(1)\end{array}$ & $\begin{array}{l}4,7 \\
(6)\end{array}$ & - & $\begin{array}{c}2 \\
(7)\end{array}$ & $\begin{array}{c}1 \\
\text { (7) }\end{array}$ & $\begin{array}{l}2,2 \\
(2)\end{array}$ \\
\hline $50-60$ & $\begin{array}{l}1,3 \\
(2)\end{array}$ & $\begin{array}{l}1,1 \\
(2)\end{array}$ & $\begin{array}{l}1,4 \\
(2)\end{array}$ & $\begin{array}{l}3,6 \\
(5)\end{array}$ & $\begin{array}{l}0,4 \\
\text { (1) }\end{array}$ & $\begin{array}{l}0,8 \\
(7)\end{array}$ & & $\begin{array}{l}11,4 \\
(13)\end{array}$ & $\begin{array}{l}2,4 \\
(3)\end{array}$ & & - & $\begin{array}{c}1 \\
(1)\end{array}$ & $\begin{array}{c}2,8 \\
(68)\end{array}$ \\
\hline $60-70$ & - & $\begin{array}{c}3,9 \\
(11)\end{array}$ & $\begin{array}{l}4,1 \\
(4)\end{array}$ & $\begin{array}{l}2,6 \\
(3)\end{array}$ & - & - & & - & - & & & - & $\begin{array}{l}1,9 \\
(31)\end{array}$ \\
\hline $70-80$ & & $\begin{array}{c}4 \\
(5)\end{array}$ & $\begin{array}{l}2,5 \\
(2)\end{array}$ & $\begin{array}{l}3,4 \\
(2)\end{array}$ & $\begin{array}{c}1 \\
(1)\end{array}$ & - & & & & & & & $\begin{array}{l}0,8 \\
(1)\end{array}$ \\
\hline $80-90$ & & $\begin{array}{l}2,1 \\
(8)\end{array}$ & $\begin{array}{l}1,8 \\
(12)\end{array}$ & - & - & - & & & & & & & \\
\hline $90-100$ & & $\begin{array}{l}1,5 \\
(4)\end{array}$ & $\begin{array}{l}1,4 \\
(4)\end{array}$ & - & & - & & & & & & & \\
\hline $100-110$ & & $\begin{array}{c}3 \\
(4)\end{array}$ & $\begin{array}{l}3,2 \\
(7)\end{array}$ & - & & & & & & & & & \\
\hline $110-120$ & & $\begin{array}{l}3,9 \\
(6)\end{array}$ & $\begin{array}{l}3,6 \\
(8)\end{array}$ & & & & & & & & & & \\
\hline $120-130$ & & $\begin{array}{c}1,9 \\
(11)\end{array}$ & - & & & & & & & & & & \\
\hline $130-140$ & & $\begin{array}{l}3,7 \\
(7)\end{array}$ & - & & & & & & & & & & \\
\hline $140-150$ & & $\begin{array}{c}2,6 \\
(13)\end{array}$ & - & & & & & & & & & & \\
\hline $150-160$ & & $\begin{array}{c}2 \\
(8)\end{array}$ & - & & & & & & & & & & \\
\hline $160-170$ & & $\begin{array}{l}1,8 \\
(2)\end{array}$ & & & & & & & & & & & \\
\hline $170-180$ & & $\begin{array}{l}3,1 \\
(6)\end{array}$ & & & & & & & & & & & \\
\hline $180-190$ & & $\begin{array}{l}1,5 \\
\text { (1) }\end{array}$ & & & & & & & & & & & \\
\hline $190-200$ & & - & & & & & & & & & & & \\
\hline
\end{tabular}

por $\mathrm{m}^{2}$; muy baja en comparación con el promedio de 88 fragmentos por $\mathrm{m}^{2}$ en los Sondeos (1.145 en total). Solo en el Sondeo V, ubicado en uno de los sectores de mayor concentración de artefactos en superficie, se mantiene en los primeros $10 \mathrm{~cm}$ el promedio general de fragmentos en superficie $(\mathrm{n}=88)$, que disminuye en los niveles inferiores.

El peso promedio de la cerámica de la superficie general $(2,35 \mathrm{~g})$ es superior a la media del primer nivel (hasta $10 \mathrm{~cm}$ ), pero en los siguientes niveles, 
la media es similar y mayor a la de la muestra de superficie, alternando con niveles de menor peso (Figura 5). La media de los pesos promedio de cada nivel $(\mathrm{x}=2,68, \mathrm{~S}=0,73)$ es un poco mayor a la de la superficie. Para evaluar si las muestras de superficie y de los sondeos difieren entre sí de manera significativa respecto de sus medias, se aplicó la prueba $t$ de Student, cuyo resultado $(t=1,38)$ permite sostener que no existen diferencias significativas entre los pesos promedios de los dos grupos (Hernández Sampieri et al. 1995:392-394).

Para establecer si existe correlación entre la profundidad y la cantidad y peso de la cerámica se sacó el coeficiente de correlación de Pearson para ambos casos. Se obtuvo un coeficiente de $-0,75$ $\left(r^{2}=0,57\right)$ entre la profundidad y el número de materiales, lo cual indica una "correlación negativa considerable", es decir que la cantidad de cerámica disminuye al aumentar la profundidad, mientras que entre el peso y la profundidad no existe correlación alguna $\left(r=-0,06 ; r^{2}=0,004\right)$ (Hernández Sampieri

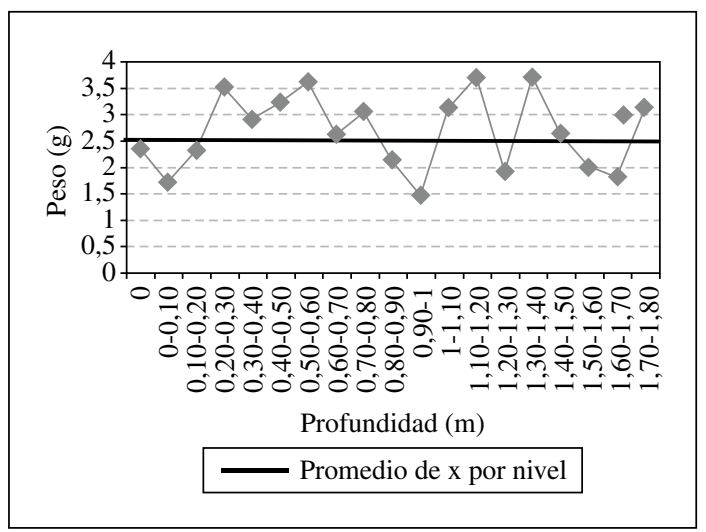

Figura 5. Agua Amarga: Promedio de peso de la cerámica de Agua Amarga por niveles de $10 \mathrm{~cm}$ de profundidad (Sondeos I a XIII).

Agua Amarga: Mean weight of potsherds by $10 \mathrm{~cm}$ levels (Test pits I-XIII). et al. 1995:392-394). En los niveles inferiores hay menor cantidad de elementos, sin embargo, son más pesados que los de los niveles superiores.

Estos datos generales fueron analizados en cada sondeo, observándose variaciones con respecto a los valores promedio obtenidos (Tabla 6). La correlación entre la profundidad y la cantidad de fragmentos se mantiene negativa en los sondeos I a V y VII excavados en el sector de Parral y la barranca sur; en tanto que en estos mismos sondeos la correlación entre la profundidad y el peso es débil en cualquier sentido (positivo o negativo). En los sondeos excavados en la barranca norte, donde no se registran operaciones agrícolas, los valores de la correlación varían en ambos casos, en el sentido de que a mayor profundidad hay mayor cantidad de artefactos (correlación positiva débil o intermedia) y mayor peso (aunque los valores de la correlación tampoco son importantes).

Al comparar la frecuencia de fragmentos por categorías de peso (Figuras 6 y 7 y Tabla 7) observamos

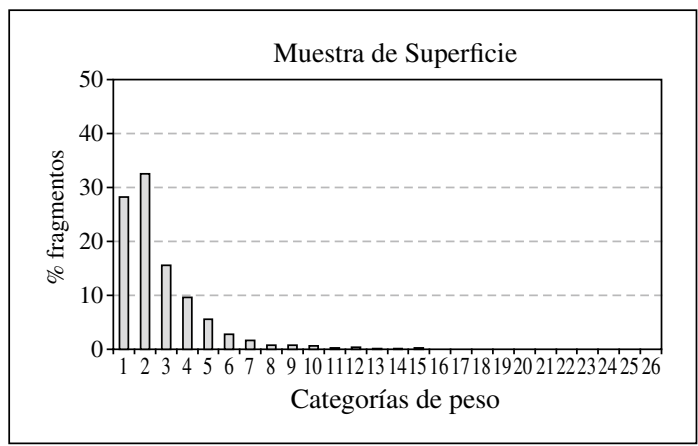

Figura 6. Frecuencia de fragmentos de cerámica por categoría de peso (Muestra de Superficie).

Frequency of potsherds by weight category (Surface sample).

Tabla 6. Coeficiente de Correlación de Pearson (Profundidad-número de artefactos; Profundidad-peso de artefactos) por sondeo. Pearson Coefficient of Correlation (Depth-artifacts number; depth artifacts weight) by Test pit.

\begin{tabular}{lccccccccccccc}
\hline Sondeo & I & II & III & IV & V & VI & VII & VIII & IX & X & XI & XII & XIII \\
\hline Prof/N & $-0,83$ & $-0,49$ & $-0,73$ & $-0,43$ & $-0,85$ & 0,45 & -1 & 0,22 & 0,04 & 0,35 & 0,34 & $-0,93$ & 0,28 \\
Prof/Peso & $-0,65$ & $-0,26$ & 0,28 & $-0,2$ & $-0,54$ & 0,16 & $-0,24$ & 0,64 & 0,57 & 0,99 & $-0,23$ & $-0,31$ & $-0,65$ \\
\hline
\end{tabular}




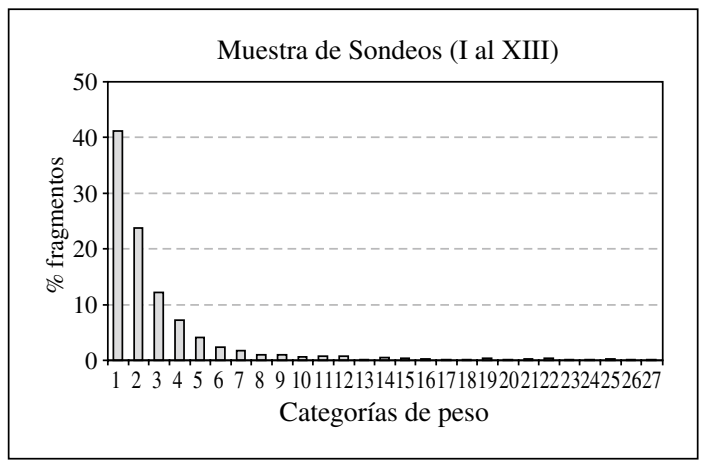

Figura 7. Frecuencia de fragmentos de cerámica por categoría de peso (muestra de Sondeos I al XIII).

Frequency of potsherds by weight category (sample of Test pits I-XIII).

Tabla 7. Categorías de peso de la cerámica de Agua Amarga. Category of pottery sherds weight from Agua Amarga.

\begin{tabular}{crr}
\hline $\begin{array}{c}\mathrm{N}^{\circ} \text { en las } \\
\text { Figuras } 6 \text { y } 7\end{array}$ & $\begin{array}{r}\text { Superficie } \\
\text { (peso g) }\end{array}$ & $\begin{array}{r}\text { Sondeo } \\
\text { (peso g) }\end{array}$ \\
\hline 1 & $0-0,99$ & $0-0,99$ \\
2 & $1-1,99$ & $1-1,99$ \\
3 & $2-2,99$ & $2-2,99$ \\
4 & $3-3,99$ & $3-3,99$ \\
5 & $4-4,99$ & $4-4,99$ \\
6 & $5-5,99$ & $5-5,99$ \\
7 & $6-6,99$ & $6-6,99$ \\
8 & $7-7,99$ & $7-7,99$ \\
9 & $8-8,99$ & $8-8,99$ \\
10 & $9-9,99$ & $9-9,99$ \\
11 & $10-10,99$ & $10-10,99$ \\
12 & $11-11,99$ & $11-11,99$ \\
13 & $12-12,99$ & $12-12,99$ \\
14 & $13-13,99$ & $13-13,99$ \\
15 & $14-14,99$ & $14-14,99$ \\
16 & $15-15,99$ & $15-15,99$ \\
17 & $17-17,99$ & $16-16,99$ \\
18 & $19-19,99$ & $17-17,99$ \\
19 & $21-21,99$ & $18-18,99$ \\
20 & $22-22,99$ & $19-19,99$ \\
21 & $23-23,99$ & $20-20,99$ \\
22 & $24-24,99$ & $21-21,99$ \\
23 & $28-28,99$ & $22-22,99$ \\
24 & $35-35,99$ & $23-23,99$ \\
25 & $38-38,99$ & $24-24,99$ \\
26 & 71,73 & $25-25,99$ \\
27 & & $26-26,99$ \\
\hline
\end{tabular}

que en ambas muestras el $76 \%$ de los fragmentos pesan menos de $3 \mathrm{~g}$. El $64 \%$ de los fragmentos recuperados en los sondeos pesaban menos de $2 \mathrm{~g}$ y la mayoría de éstos ( $41 \%$ del total), inclusive menos de $1 \mathrm{~g}$. Si bien la distribución en la muestra de superficie es similar $(60 \%$ de los fragmentos menores a $2 \mathrm{~g}$ ), hay más cantidad de fragmentos que pesan entre 1 y $2 \mathrm{~g}$ (aproximadamente $33 \%$ sobre el total), y entre 2 y $3 \mathrm{~g}$ ( $12 \%$ en sondeos, $16 \%$ en superficie). Aunque se observa mayor frecuencia de fragmentos más pesados en la superficie que en los sondeos, esta diferencia es mínima.

Luego se analizó la representación de los distintos "tipos" de cerámica identificados a partir de los fragmentos decorados. La proporción de cerámica decorada en la muestra de superficie es del $12,4 \%$ del total, y se distribuyen como sigue: $0,7 \%$ de la muestra (13 fragmentos) del tipo gris y marrón inciso; 11,27\% (208 tiestos) naranja alisado pulido y/o pintado, y rasgos decorativos propios de la cerámica Diaguita-Inca en 8 fragmentos $(0,4 \%$ sobre el total de la muestra).

Si comparamos las frecuencias de fragmentos de los tres tipos de decoración representados (Tabla 8 y Figura 8) notamos que la mayor cantidad de tiestos identificables en la superficie y en los sondeos son Viluco y Viluco Inca, aunque hay una notable distinción entre la superficie y los sondeos, ya que en el segundo caso disminuye la diferencia con los otros tipos. La mayor cantidad de fragmentos con estas características registrados en la excavación se encontraba en los niveles superiores (hasta $30 \mathrm{~cm}$ ): los pesos promedios comparados en la Tabla 8 pueden equipararse a la relación general de pesos entre la superficie y los primeros $10 \mathrm{~cm}$ de profundidad (Tabla 4). La misma relación de pesos entre ambas muestras se puede establecer con los fragmentos Diaguita Chileno Inca, registrados en la superficie y en los primeros $10 \mathrm{~cm}$. Los tiestos grises con decoración incisa de mayor peso se encontraron en

Tabla 8. Peso de los distintos tipos de cerámica de las muestras de superficie y sondeos I al XIII de Agua Amarga. Different pottery types weight in surface and test pits samples I to XIII from Agua Amarga.

\begin{tabular}{ccccccc}
\hline Tipo & \multicolumn{2}{c}{ Gris inciso } & \multicolumn{2}{c}{ Naranja } & \multicolumn{2}{c}{ Rojo } \\
\hline \multirow{2}{*}{ Procedencia } & Superf. & Sondeo & Superf. & Sondeo & Superf. & Sondeo \\
$\mathrm{n}$ & 12 & 17 & 205 & 48 & 8 & 5 \\
Peso Total & 27,18 & 104,55 & 594,5 & 83,52 & 17,91 & 4,63 \\
$\mathrm{x}$ & 2,3 & 6,16 & 2,9 & 1,74 & 2,24 & 1,16 \\
$\mathrm{~S}$ & 0,02 & 5,8 & 3,8 & 2,55 & 1,25 & 0,96 \\
\hline
\end{tabular}




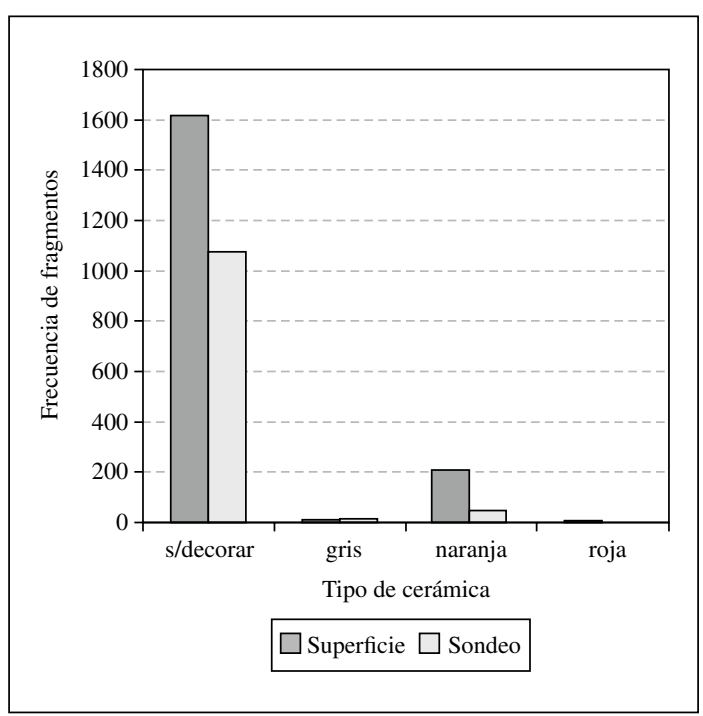

Figura 8. Frecuencia de fragmentos de cerámica por "tipos" en las muestras de superficie y sondeos (I al XIII).

Frequency of potsherds by type categories in surface collections and Test pit samples (I-XIII).

los niveles más profundos y, como se observa en la Tabla 8, la proporción de cerámica gris es mayor en niveles más profundos que en la superficie.

El peso promedio de la cerámica de los tres tipos comparados en la superficie es similar, pero la diferencia entre el peso de los tiestos grises y los pintados en los niveles inferiores es notable, porque además de aumentar considerablemente el de los primeros, disminuye el de los segundos.

La baja representación de la cerámica incisa en la superficie no responde a una débil presencia en el registro, ya que comprobamos en las excavaciones que este componente se encuentra en los niveles inferiores que no han sido alcanzados por la actividad agrícola. En tanto que la proporción de cerámica Diaguita-Inca es muy baja (menos del 1\%) en ambas muestras, siendo probablemente más representativa de la proporción original de este componente en el sitio.

\section{Consideraciones Finales y Conclusiones sobre los Alcances de la Investigación Arqueológica de los Conjuntos Cerámicos de Zonas Aradas}

La metodología de muestreo de tipo probabilístico sistemático ofreció ventajas y limitaciones para el abordaje del sitio: permitió identificar los límites y el tamaño de la dispersión de materiales arqueológicos en la finca, establecer que la misma presenta una distribución de tipo agrupada y reconocer además las áreas donde hay mayor concentración de materiales. Notamos que las concentraciones de cerámica se dispersan en relación con la dirección de las operaciones de labranza y de la pendiente.

Observamos que el sitio adquiere la forma del espacio agrícola superpuesto, y los límites y la superficie se confunden con las sucesivas intervenciones agrícolas. La superficie de Agua Amarga multiplica varias veces la de otros sitios del área que no se encuentran sometidos a este tipo de alteración; por ejemplo, los sitios multicomponentes del piedemonte del sur de San Juan no superan las 20 ha (Cahiza 2003). El tamaño notable de la superficie de distribución de material arqueológico debe ser atribuido a los factores posdepositacionales comentados.

Las limitaciones del método son mayores para determinar el número de concentraciones y las agrupaciones intrasitio, ya que no es posible la identificación de las superficies, los límites y formas de las mismas. La posibilidad de ubicar áreas de actividad o patrones de uso del espacio en un terreno tan modificado está además restringida por la escasa visibilidad de los conjuntos.

Aunque se identificaron los sectores donde hay mayor concentración de materiales, es dificultoso realizar excavaciones extensas en un terreno privado que se encuentra cultivado. Algunos de los sondeos de prospección pudieron excavarse dentro del parral. En uno de los sondeos (V), ubicado en uno de los sectores de mayor concentración de cerámica, se observa correspondencia entre la mayor densidad de materiales en superficie y en los niveles inferiores, por lo que podría considerarse la distribución de la cerámica en superficie para localizar sectores de mayor concentración. En el parral, los niveles superiores de los sondeos se encuentran modificados por las operaciones de labranza mecánica. Mediante la excavación, y de acuerdo a lo que suponíamos teniendo en cuenta la profundidad en que actúan las herramientas más perturbadoras, como el arado, comprobamos que las operaciones agrícolas ejecutadas en Agua Amarga han transformado los primeros 30 ó $35 \mathrm{~cm}$, incidiendo en la integridad del registro y la fragmentación de los artefactos.

En los sondeos ubicados sobre la margen izquierda del arroyo Agua Amarga, donde no se registran operaciones agrícolas, los artefactos presentan pesos 
más altos en aquellos niveles, denotando una mejor conservación.

El grado de modificación del sitio se manifiesta en la baja densidad de los materiales, y en los pesos de los mismos. Boismier (1997) y Diez Martín (2003) destacaban la progresiva disminución de la densidad de los materiales en superficie, lo cual es una consecuencia, entre otros factores, del "incremento del tamaño de los sitios". La tendencia según estos estudios es que los fragmentos de mayor tamaño (peso en este caso) estén representados exageradamente en la superficie. En Agua Amarga, y según los análisis realizados, no existe una diferencia significativa entre el promedio de peso de los fragmentos enterrados y los de la superficie. Sin embargo, en comparación con los pesos medios de los tiestos de otros sitios menos impactados del área (v.gr.: $\mathrm{x}=3,77$ para Retamito, San Juan (Cahiza 2003) las cifras obtenidas en Agua Amarga son muy bajas.

Estos resultados sugieren que en Agua Amarga actuarían sobre la composición de los conjuntos de la superficie y los primeros niveles dos tipos de procesos culturales señalados por Lewarch y O'Brien (1981:305): la labranza y la "perturbación ocupacional" (occupational disturbance). El primero de estos procesos provoca el desplazamiento vertical de los materiales más pesados, y posteriormente el pisoteo produce una mayor fragmentación en la cerámica de superficie, promoviendo el enterramiento de los fragmentos más pequeños, lo cual denota el peso promedio del primer nivel de excavación, más bajo que el de la superficie.

Las características de alta fragmentación de los artefactos (mayor cantidad y menor peso en los primeros niveles), disminución de la densidad y gran dispersión de los materiales en superficie con límites indefinidos de las concentraciones coinciden con los criterios que Boismier (1997) estableció para determinar que el sitio se encuentra en un grado de alteración intermedio o avanzado.

Sin embargo, sostenemos que en sitios con estas características es posible el estudio de las ocupaciones humanas a partir de los materiales, en este caso, de la cerámica. Mediante la recuperación intensiva y sistemática del componente cerámico y su análisis, se identificó en Agua Amarga la secuencia característica del proceso cultural regional. Se contrastó la secuencia de las ocupaciones a través de las excavaciones, identificando un nivel sin intrusiones ni alteraciones correspondiente al componente temprano-medio, mientras que el nivel tardío se encuentra muy impactado por la labranza, entre otros factores. A priori puede concluirse que se ha alterado la estructura estratigráfica original, como concluían otros trabajos, porque todos los tipos se encuentran representados en la superficie; pero al compararlo con la excavación se observa que la proporción de los materiales del componente temprano-medio no es representativa de esta ocupación en cuanto a cantidad y peso. La proporción de cerámica del tardío, y su peso promedio, es mayor en la superficie, en tanto que la proporción de cerámica Diaguita Chileno Inca es en todos los casos muy baja, probablemente por ser muy baja la frecuencia original de este tipo.

Otros aspectos considerados fueron el tipo y la intensidad de las ocupaciones y actividades que se han desarrollado en el sector. La información obtenida sobre los usos del espacio en tiempos históricos mediante documentos, la consulta de otros estudios, la comunicación personal con los propietarios y operarios y la observación directa de las operaciones agrícolas, demuestra que las transformaciones principales del sitio se produjeron durante los últimos 30 años, lo cual se evidencia en el registro, ya que aparte de los materiales indígenas, sólo se reconocen artefactos modernos asociados a dichas labores (alambres, fragmentos de mangueras, botellas, bolsas de plástico).

Por otro lado, el estudio del sitio se ha emprendido dentro del enfoque regional y diacrónico dado a las investigaciones del área (Ots 2006, 2007). De esta manera, puede considerarse el criterio de ordenamiento tecnotipológico de la cerámica como alternativa para establecer la secuencia de ocupaciones del sitio, tomando como parámetro otros contextos fechados en la región y contrastándola mediante las excavaciones.

Consideramos que estas conclusiones sobre el efecto de las operaciones agrícolas sobre los conjuntos cerámicos fundamentan la decisión de no desestimar la investigación de sitios arqueológicos del Valle de Uco que se encuentran en estas condiciones, y asimismo en otras áreas de similares características, a pesar de presentar mayores dificultades que los sitios que no han sido ocupados tan intensamente. Los datos presentados sugieren que la investigación de este tipo de sitios permitirá resolver algunos problemas (en este caso, tecnológicos o cronológicos), en tanto que estará limitada en otros 
aspectos (identificación de áreas de actividad, uso del espacio, funcionalidad, contextos funcionales, entre otros).

El estudio que realizamos en un "área arada" nos permite arribar a conclusiones que en algunos casos coinciden y en otros disienten con las de otros trabajos en este tipo de sitios arqueológicos o experimentales. Probablemente, las diferencias son consecuencia de la combinación de distintas variables además de la labranza, ya que otros procesos que inciden -como el riego y el pisoteo- no se han tenido en cuenta en este tipo de trabajos.
Agradecimientos: A los Sres. Ramón y José Hinojosa, propietarios de la Finca Agua Amarga. Al Dr. Roberto Bárcena y al equipo de investigación que dirige, por su apoyo. A los Sres. Gustavo Figueroa, Lucas Páez, Juan Pablo Aguilar, Lourdes Iniesta y Marcos Forgeaux, alumnos de la carrera de Historia (Facultad de Filosofía y Letras de la Universidad Nacional de Cuyo), por su colaboración en las tareas de campo. Al Ingeniero Agrónomo David Boulet. A la Dra. Ángela De Blasis (Área de Ciencias Exactas, CRICYT). A los evaluadores y editores de Chungara, cuyas observaciones y sugerencias me permitieron mejorar este artículo.

\section{Referencias Citadas}

Baker, Ch.M.

1978 The size effect: an explanation of variability in surface: artifact assemblage content. American Antiquity 43:288-293.

Bárcena, J.R.

1998 Arqueología de Mendoza. Las Dataciones Absolutas y sus Alcances. Ediunc, Mendoza.

2001 Prehistoria del Centro-Oeste argentino. En Historia Argentina Prehispánica Tomo 2, compilado por E. Berberián y A. Nielsen, pp. 561-627. Brujas, Córdoba.

Bárcena, J.R. y A. Román

1990 [1986-87] Funcionalidad diferencial de las estructuras del Tambo de Tambillos. Anales de Arqueología y Etnología 41-42:7-81.

Boismier, W.A.

1997 Modelling the effects of tillage processes on artefact distributions in the ploughzone. A simulation study of tillageinduced pattern formation. BAR, British Series 259.

Cahiza, $P$.

2003 La Dominación Inca en las Tierras Bajas de Mendoza y San Juan. Tesis doctoral inédita, Facultad de Filosofía y Letras, Universidad Nacional de Cuyo, Mendoza.

Canals Frau, S.

1956 Algunos aspectos de la cultura de Agrelo (Prov. de Mendoza). Anales de Arqueología y Etnología 12:7-18.

Canals Frau, S. y J. Semper 1956 La cultura de Agrelo (Mendoza). Runa II (2):169-187.

Civalero de Biset, M.T.

1991 Modificaciones en los filos de artefactos líticos a partir de una experiencia de pisoteo. Shincal 3. Publicación Especial en Adhesión al X Congreso Nacional de Arqueología Argentina Tomo 3:200-205.

De Luca, L. y S. Soria 1995 Geografía física: relieve e hidrografía. En Tunuyán, Historia y Perspectiva, compilado por P. Lacoste, pp. 26-27. UNO, Mendoza.

Diez Martín, F.

1998 Observaciones sobre la incidencia del laboreo agrícola en los agregados líticos. El experimento de Las Cuestas (Tudela del Duero, Valladolid). Boletín del Seminario de Estudios de Arte y Arqueología (BSAA) LXIV:29-40.
2003 Las alteraciones inducidas por el laboreo agrícola: la influencia del movimiento vertical en los yacimientos paleolíticos de los páramos de Montemayor-Corcos (Valladolid y Burgos). ZEPHYRVS, Revista de Prehistoria y Arqueología LVI:49-60.

Dunnell, R.C. y J. Simek

1995 Artifact size and plowzone processes. Journal of Field Archaeology 22:305-319.

Gutiérrez de Manchón, M.J. y ME. Furlani de Civit

1997 Decisiones empresariales y fragilidad ambiental. En Mendoza: Una Geografía en Transformación II, compilado por M.E. Furlani de Civit y M.J. Gutiérrez de Manchón, pp. 233-243. Facultad de Filosofía y Letras, Universidad Nacional de Cuyo, Mendoza

Hermosilla, N., B. Saavedra, J. Castelleti y L. Quiroz 2003 El prodigioso Estero de Lo Campo: estudio de sitios arados en el curso superior del río Aconcagua, V Región, Chile. Actas del XIII Congreso Nacional de Arqueología Argentina, Tomo 3:217-230. Brujas, Córdoba.

Hermosilla, N., C. González y D. Baudet

2005 Sitio Peldehue: Rescate de un contexto funerario inka en un sitio habitacional Aconcagua. Xama 15-18:263-278.

Hernández Sampieri, R., C. Fernández Collado y P. Baptista Lucio

1995 Metodología de la Investigación. McGraw-Hill, Colombia.

Hodder, I. y C. Orton

1990 [1976] Análisis Espacial en Arqueología. Traducido por M.J. Aubet y M. Tenas. Crítica, Barcelona.

Hunt, D.

1983 Maquinaria Agrícola. Rendimiento Económico, Costos, Operaciones, Potencia y Selección de Equipo. Limusa, México.

Lagiglia, $\mathrm{H}$.

1978 La cultura de Viluco del Centro Oeste argentino. Actas y Memoria del IV Congreso Nacional de Arqueología Argentina (Primera Parte). Revista del Museo de Historia Natural de San Rafael (Mendoza), Tomo 3 (1/4): 227-265.

Laguens, A.

1993-1994 Observación controlada y análisis estadístico de procesos de formación de sitio en el Árido del centro 
de Argentina. Relaciones de la Sociedad Argentina de Antropología, Tomo XIX: 215-255.

Lewarch, D.E. y M.J. O'Brien

1981 The expanding role of surface assemblages in archaeological research. En Advances in Archaeological Method and Theory 4, editado por M. Schiffer, pp. 297-334. Academic Press, Tucson, Arizona.

Michieli, C.

1998 Aproximaciones a la identificación de una cerámica indígena posthispánica del sur de San Juan. Publicaciones 22:55-76.

Nielsen, A.

1991 Se hace camino al andar. Estudio experimental sobre patrones de fractura en material cerámico sometido a pisoteo. Shincal, 3. Publicación Especial en Adhesión al X Congreso Nacional de Arqueología Argentina, Tomo 1:116-139.

Odell, g H. y F. Cowan

1987 Estimating tillage effects on artifact distributions. American Antiquity 52:456-487.

Orton, C., P. Tyers y A. Vince

1997 [1993] La Cerámica en Arqueología. Traducido por R. Barceló y J. A. Barceló. Crítica, Barcelona.

Ots, M.J.

2006 Prospección arqueológica de la cuenca del río De las Tunas (dptos. Tupungato- Tunuyán, Mendoza). Cuadernos 29:43-60, Jujuy.

Notas

1 Proyecto "Arqueología y Etnohistoria incaica del Centro Oeste argentino", PIP 2026, CONICET, dirigido por el Dr. Roberto Bárcena.

2 Tesis doctoral sobre "La presencia inca en el Valle de Uco" (Ots 2007) que desarrollamos bajo la dirección del Dr. Roberto Bárcena.

3 Observación y comunicación personal con el propietario de la finca, sr. Ramón Hinojosa, el encargado, sr. Domingo Hurtado y el Ingeniero agrónomo David Boulet.

4 La superficie del espacio cultivado es de 52,3 ha. El muestreo del $5 \%$ de las transectas cubrió una superficie de 1,9 ha, el $3,6 \%$ de la superficie total.

5 Otras cuestiones están siendo evaluadas en una Unidad de Observación Controlada (UOC, siguiendo la propuesta de Laguens (1993-1994)) dentro del espacio cultivado en Agua
2007 La Presencia Incaica en el Valle de Uco, Mendoza. Tesis doctoral, Facultad de Filosofía y Letras, Universidad Nacional de Cuyo, Mendoza.

Planella, M.T. y R. Stehberg

1997 Intervención Inca en un territorio de la cultura local Aconcagua de la zona centro-sur de Chile. Tawantinsuyu 3:58-78.

Prieto, M. del R.

2000 Formación y consolidación en un área marginal del Reino de Chile: La provincia de Cuyo en el siglo XVII. Tesis doctoral, publicada en Anales de Arqueología y Etnología, 52-53.

Roper, D. C.

1976 Lateral displacement of artifacts due on plowing. American Antiquity 41:372-375.

Rusconi, C.

1962 Poblaciones Pre y Posthispánicas de Mendoza, Vol. III, Arqueología. Gobierno de Mendoza, Mendoza.

Shennan, S.

1992 [1988] Arqueología cuantitativa. Traducido por J A. Barceló. Crítica, Barcelona.

Studdert, G.

2001 Labranza conservacionista. (10 marzo) www.inta.gov. ar/balcarce/info/documentos/recnat/suelos/labranzacons. htm (15 febrero 2006).

Amarga, considerando las operaciones propias de este tipo de cultivos en la región. Los datos analizados hasta el momento permiten sostener que el desplazamiento horizontal sigue una dirección levemente inclinada con respecto a la de la maquinaria y no supera $l o s ~ 40 \mathrm{~cm}$ en artefactos de cualquier tipo y tamaño; se mantiene una distribución "agrupada" de los artefactos, concentrándose sobre todo en los sectores laterales, los más elevados de la cuadrícula; no se observan variaciones anuales significativas en cuanto al tamaño de los artefactos.

6 Junto a las piezas descritas por Lagiglia (1978) en la clasificación de la facie II de Viluco, se expone en el Museo Municipal de Historia Natural de San Rafael bajo el rótulo de Agua Amarga, un aribaloide cuya decoración (pintura roja sobre la superficie naranja alisada) corresponde a este tipo. 